We recently completed a study, similar to that by Bhandari, ${ }^{1}$ exploring the readability of assessment letters being produced by an adult community mental health team (CMHT) in south-west England. We looked at all new assessment letters produced over a 3-month period. As the CMHT assessment is usually the first point of contact with services, we felt that the readability of assessment letters was particularly important with regard to engagement and promoting a shared understanding of a person's difficulties.

We used readability software available as a standard with Microsoft Word 2007 to establish the Flesch Reading Ease. ${ }^{2}$ This is a validated tool widely used to assess readability, based on the syllabic and sentence structure of the text. Reading ease on this scale ranges from 0 to 100 , with specific intervals categorised from 'very easy' (90-100) to 'very difficult' (0-29).

Like Bandari, we found that no letters were 'easy' or 'very easy' to read. However, we found that letters were significantly more readable $(P=0.004)$ if they were addressed to the patient with the general practitioner copied in, rather than vice versa. We speculate that this is because when dictating a letter to the patient, the patient and their understanding is borne in mind to a greater extent than when addressing a colleague.

In addition, the readability of letters varied by professional group. Whereas there was no significant difference in readability between junior doctors', occupational therapists' and social workers' letters, community mental health nurses and consultants produced significantly less readable letters $(P=0.001$ and $P=0.000$ respectively). The fact that no letters reached the standard of 'easy' or 'very easy' may reflect the difficulty of using simple terms to describe psychopathology. However, some authors produced much more readable letters than others, which suggests that improvement is possible.

We found it interesting that junior doctors wrote more readable letters than their consultant colleagues. We speculated that corresponding directly with patients is a skill with which consultants may lack historical experience as they have spent more of their careers corresponding principally with fellow health professionals. As a result they may be less familiar with methods to make letters more readable to the general public.

We concluded that assessment letters produced by community mental health workers do not score well for readability. We feel is of the utmost importance that the reading ability of our patients is borne in mind when writing such letters. Simple changes such as addressing the patient directly may help improve readability.

1 Bhandari N. Readability - writing letters to patients in plain English. Psychiatrist 2010; 34: 454.

2 Flesch R. The Art of Readable Writing. Harper \& Row, 1973.

Elizabeth A. O'Mahony specialty registrar, Avon and Wiltshire Mental Health Partnership NHS Trust, Bristol, email: elizabeth.omahony@nhs.net, Nicola J. Kalk clinical research fellow, Imperial College London.

doi: $10.1192 / p b .35 .1 .30 b$

\section{Risk to staff in a crisis resolution team}

Crisis resolution and home treatment (CRHT) teams are now well established. There is significant evidence that they reduce bed use, are cost-effective and patients prefer them to admission. ${ }^{1}$

A CRHT team is dependent on the expertise and imagination of its staff to help understand and resolve a crisis. However, the risk to staff of working intensively with people who would otherwise be in hospital is not well documented. The evaluations of CRHTs have not considered the staff or the impact of frequent visits from different staff on people at high risk of acting violently. ${ }^{1,2}$ Risk management is a continuous task in a CRHT team. There is some concern that risks to patients may increase with the introduction of a CRHT team, although this is far from established. ${ }^{3}$

We conducted an anonymous survey of the Hammersmith and Fulham Crisis Resolution Team in London. We asked whether they had felt physically vulnerable during community visits and encouraged them to describe any relevant incidents. Respondents included doctors, nurses, occupational therapists, support workers and bank staff. Duration of work with the team ranged from a few weeks to over 6 years. All had at least two jobs in psychiatric services before joining the crisis team and most had several years of previous mental health experience, in CRHT teams and on wards.

More than half of the respondents (13 of 20) had felt physically vulnerable while on a home visit. Their experiences ranged from feeling concerned about personal safety when with patients who were aroused or were experiencing psychosis, to being chased out of an abode when violence was threatened. No one had been physically harmed. The remaining seven people had all worked with the team for less than a year. Everyone working in the team for longer than a year reported feeling physically vulnerable during at least one visit.

We found that exposure to risk from patients was ubiquitous among all established CRHT staff in our study. It is particularly important to document risk to staff to avoid minimisation. As health services reduce costs, crisis teams will be asked to increase the threshold and reduce the duration of in-patient care further. Crisis resolution home treatment teams receive several hundred referrals a year. The short response times, high expectations and anxiety of referrers, as well as pressure to act and prevent admission all potentially reduce thinking about risk.

1 Joy $C B$, Adams CE, Rice K. Crisis intervention for people with severe mental illnesses. Cochr Dat Syst Rev 2007; issue 4. CD001087.

2 National Audit Office. Helping People through Mental Health Crisis: The Role of Crisis Resolution and Home Treatment Services. TSO (The Stationery Office), 2007

3 Tyrer P, Gordon F, Nourmand S, Lawrence M, Curran C, Southgate D, et al. Controlled comparison of two crisis resolution and home treatment teams. Psychiatrist 2010; 34: 50-4.

Nicky Goater consultant psychiatrist, Hammersmith and Fulham Crisis Resolution Team, West London Mental Health Trust, London, email: nicky.goater@wlmht.nhs.uk, Eleanor Rowland medical student, Imperial College London.

doi: $10.1192 / p b .35 .1 .31$

\section{Polypharmacy: should we or shouldn't we?}

Much has been written recently in The Psychiatrist about how psychiatrists should manage antipsychotic polypharmacy. Taylor ${ }^{1}$ could hardly be more emphatic: 'evidence supporting antipsychotic polypharmacy has, if anything, diminished and 
evidence suggesting or demonstrating harm has grown'. He concludes that 'mounting awareness of the probable futility of antipsychotic polypharmacy is reflected in the latest guidance issued by the National Institute for Health and Clinical Excellence'.

Lepping \& Harbone ${ }^{2}$ draw attention to the need for a 'more balanced view with regard to polypharmacy in a patient group that is often non-responsive'. We would like to address issues raised by Odelola \& Ranceva. ${ }^{3}$

First, Odelola \& Ranceva speculate that the persistence of antipsychotic polypharmacy despite repeated guidance against it may indicate that this is one area where clinical practice is ahead of research evidence. They reiterate Lepping \& Harbone's point that in the case of polypharmacy the evidence provides no support either way - hardly a ringing endorsement. Additionally, they praise the excellent recommendations by Langan \& Shajahan. ${ }^{4}$ In the context of their letter we would be concerned that this is potentially misleading. Langan \& Shajahan urge extreme caution if one uses polypharmacy, supported by thorough explanatory documentation, rigorous monitoring and ongoing review. They conclude with the caveat that the 'worrying relationship' between the use of polypharmacy and mortality merits investigation and that it remains 'more art than science'. The message to take home seems to be 'avoid if possible'.

Among the routes to antipsychotic polypharmacy, nearly all of the researchers quoted here identify the failure to complete a switch from one agent to the other as a starting point for polypharmacy - this surely represents an opportunity for psychiatrists to tackle unplanned and inappropriate polypharmacy. The risks of high-dose prescribing should also be borne in mind.

The fact that there are probably increasing rates of polypharmacy prescribing should not be misinterpreted as evidence in support of it - once it was doubted by many that the world was spherical! Evidence suggests that the two polypharmacy scenarios outlined in the National Institute for Health and Clinical Excellence guidelines, ${ }^{5}$ cross-tapering and adding an antipsychotic to clozapine, appear reasonable. Outside these scenarios the risks $v$. benefits demand serious concern. We would echo Odelola \& Ranceva's call to be openminded about polypharmacy. This would extend to entertaining the possibility that the practice should be jettisoned in many cases. To cope with any overwhelming feelings of therapeutic nihilism, we would direct readers to Williams et al's editorial. ${ }^{6}$

1 Taylor D. Antipsychotic polypharmacy - confusion reigns. Psychiatrist 2010; 34: 41-3.

2 Lepping P, Harbone GC. Polypharmacy: how bad are we really? Psychiatrist 2010; 34: 208-9.

3 Odelola D, Ranceva N. Polypharmacy: saint or sinner? Psychiatrist 2010; 34: 354

4 Langan J, Shajahan P. Antipsychotic polypharmacy: review of mechanisms, mortality and management. Psychiatrist 2010; 34: 58-62.

5 National Institute for Health and Clinical Excellence. Schizophrenia: Core Interventions in the Treatment and Management of Schizophrenia in Primary and Secondary Care (Update). NICE, 2009.

6 Williams L, Newton G, Roberts K, Finlayson S, Brabbins C. Clozapineresistant schizophrenia: a positive approach. Br J Psychiatry 2002; 181 $184-7$

Rajesh Rajpal Regional North East GP Training Scheme, Elizabeth Owens senior registrar, National Higher Training Scheme, MacDara McCauley consultant psychiatrist, St Brigid's Hospital, Ardee, Co. Louth, Ireland, email: macdara.mccauley@hse.ie

doi: 10.1192/pb.35.1.31a

\section{Patient satisfaction rating scales $v$. patient-related outcome and experience measures}

We were pleased to read the paper by Hansen et al detailing their validation of a patient satisfaction rating scale. This sort of work is very much in keeping with the recent government paper, Equity and Excellence: Liberating the NHS. ${ }^{2}$ However, we thought that the focus of the questionnaire was too narrow: it essentially only dealt with the interaction between psychiatrist and service user in an out-patient setting. A far broader perspective would need to be taken for this instrument to be used as a service satisfaction questionnaire, because patients interact with a far greater range of people and systems as they move through a given care pathway. Even in a fairly circumscribed setting such as out-patient setting service users deal with appointment letters, receptionists, the physical environment of the waiting room, etc., even before they get to meet a psychiatrist. However, we did think that the questionnaire would make an excellent instrument for psychiatrists (and other mental health professionals) to use as part of their annual appraisal or multisource feedback, as it provides good information about the vital interaction between doctor and patient.

More pertinent to today's clinical practice are Patient Reported Outcome Measures (PROMs) and Patient Reported Experience Measures (PREMs). They provide richer information than patient satisfaction questionnaires, which are concerned with a relatively narrow (but obviously very important) area. It is possible for a user to have a satisfactory experience of a service (and score a satisfaction questionnaire highly) but a poor clinical outcome (which would not be identified by a satisfaction scale). On the other hand, PROMs and PREMs will capture not only the patient experience/ satisfaction but also the outcome from the patient's perspective. These data complement the gathering of routine clinical outcome data, which in the UK pertain primarily to the Health of the Nation Outcome Scales. ${ }^{3}$

Patient Reported Outcome Measures and Patient Reported Experience Measures have been established in acute trusts for quite so time now. They suit certain specialties well, for example post-hip operation PROMs are ubiquitous, but in mental health they are much rarer. To attempt to address this gap, we (and other colleagues from our trust) are in the process of validating a PROM/PREM for mental health service users. ${ }^{4}$ A version of the instrument specifically for use with older patients has already been successfully piloted and preliminary results will shortly be published. ${ }^{5}$

We would like to congratulate Hansen et al on their work. However, we believe that outcome data supersede patient satisfaction questionnaires in contemporary National Health Service practice. The latter can easily be incorporated into the PROMs/PREMs, which additionally provide a wider range of information.

1 Hansen LK, Vincent S, Harris S, David E, Surafudheen S, Kingdon D. A patient satisfaction rating scale for psychiatric service users. Psychiatrist 2010; 34: 485-8. 